Original Article

\title{
Effects of pelvic adjustment on pelvic posture and angles of the lower limb joints during walking in female university students
}

\author{
Misuk Cho, $\mathrm{PhD}, \mathrm{PT}^{1)}$ \\ 1) Department of Physical Therapy, Korea Nazarene University: Wolbong Ro 48, Seobuk-gu, \\ Cheonan-Si, Chungcheongnam-do 330-718, Republic of Korea
}

\begin{abstract}
Purpose] This study investigated the effects of pelvic adjustment on pelvic posture and lower limb joint angles during walking in female university students. [Subjects] Thirty healthy female university students were randomly assigned to an experimental group (pelvic adjustment group, $\mathrm{n}=15$ ) and a control group (stretching group, $\mathrm{n}=15$ ). [Methods] Pelvic adjustment was performed three times on the experimental group. The control group performed three sets of pelvic muscle stretching for 15 minutes. A back mapper and motion analysis equipment were used to measure pelvic posture and angles of lower limb joints for the experimental and control group. [Results] The values obtained before and after the intervention were compared. For the experimental group, the results were significantly different in terms of reduced differences in hip flexion between the left and right hips and in knee abduction between the left and right knees. Differences in pelvic position and pelvic torsion were also found in the experimental group. No significant differences in the control group were identified. [Conclusion] Pelvic adjustment affects pelvic position and torsion and this enhancement to pelvic stability decreases hip flexion and knee abduction during walking.

Key words: Pelvic adjustment, Lower extremity joint angle, Pelvic posture
\end{abstract}

(This article was submitted Dec. 7, 2015, and was accepted Jan. 8, 2016)

\section{INTRODUCTION}

Gait, which comprises movements that cannot be learned in a short time, refer to continuous and repeated movements of the body while maintaining stability. Gait also produces biomechanical changes and motor functional changes in the nervous system and the musculature ${ }^{1)}$. Each gait cycle is divided into a stance phase and a swing phase. Each stance phase consists of heel strike, foot flat, mid stance, and toe off movements. Each swing phase includes acceleration, mid swing, and deceleration ${ }^{2}$. Gait comprises complicated movements in which human nerves and skeletal muscles are used in unison. Substantial effort and practice are required for a person to perform their gait correctly ${ }^{3)}$. Normal gait is important to maintain harmonious trunk and pelvic turns. The dynamic balance of trunk muscle activities reduces unnecessary energy consumption and effectively eliminates false steps that may occur during gait ${ }^{4}$.

During gait, the lower limbs (which act as weight-bearing structures) work in conjunction with the head, the trunk, and the arms. In addition, the lower limbs provide basic movements that are necessary to move, push the body forward, and maintain stability and balance ${ }^{5)}$. These movements in the lower limbs are complex and efficient because they are completed by the combined functions of many human parts. Therefore, if one portion of the body involved in these movements is impaired, gait patterns become disorganized. The pelvis operates as a stable base for the trunk by supporting the abdomen and connecting the spine and the lower limbs ${ }^{6}$. Previous studies have explored the necessity of stability and posture of the pelvis during gait $^{7)}$. Some of these studies explored lumbopelvic stabilization and posture through bridge exercises ${ }^{8)}$. Others evaluated pos- 
ture following pelvic adjustment ${ }^{9)}$ or discussed gait variables ${ }^{10)}$. One study in particular focused on the correlation between pelvic position and differences in lower extremity joint angles during walking among female university students ${ }^{11)}$.

Few papers discuss whether the quality of gait improves following adjustments to the pelvis. In addition, few studies explored the effects of pelvic adjustment on differences in joint angles between the left and right lower limbs during gait. Therefore, this study used a back mapper and motion analysis equipment to examine the effects of pelvic adjustment on pelvic posture and differences in joint angles between the lower left and right limbs of female university students during gait. In this study, the Gonstead technique was used to adjust the pelvis. It is fundamentally a full-spine, osseous technique, emphasizing a specific high-velocity, low-amplitude thrust that corrects subluxation ${ }^{12}$.

\section{SUBJECTS AND METHODS}

This study was conducted on 30 women studying at K University in South Korea. These students were randomly divided into a pelvic adjustment group of 15 subjects and a stretching group of 15 subjects. The mean age, height, and weight of the pelvic adjustment group were $21.7 \pm 0.4$ years, $162.4 \pm 4.3 \mathrm{~cm}$, and $53.5 \pm 5.7 \mathrm{~kg}$, respectively. The mean age, height, and weight of the stretching group were $20.2 \pm 0.5$ years, $161.7 \pm 3.5 \mathrm{~cm}$, and $52.4 \pm 5.6 \mathrm{~kg}$, respectively. Independent t-tests were conducted to check for differences between the groups in terms of age, height, and weight. No statistically significant differences $(p>0.05)$ were detected, indicating that no issues related to homogeneity were present between the two groups. Those who had issues in the muscular, skeletal, or nervous system were excluded as subjects. Others who were excluded from the study included those who experienced daily pain in the lumbar region or the pelvis, those whose postures were not proper owing to burns or postoperative scars, and those who could not walk normally. The Institutional Review Board of K University approved this study. In accordance with the ethical standards of the Declaration of Helsinki, every subject understood the purpose of the study and provided written consent prior to their participation.

This study used a high-velocity, low-amplitude technique for prone positions derived from Gonstead's theory of pelvic adjustment. After having each subject assume a prone position, the posterior-inferior (PI) innominate bone was adjusted by moving it to the anterior-superior (AS) position. The AS innominate bone was moved to the PI position. The therapist's hands, which were overlapping, were placed on the posterior-superior iliac spine or the ischial tuberosity. Then, pressure was applied three times using gravity, acceleration, and the therapist's body weight ${ }^{13,14)}$.

Pelvic postures and differences in joint angles between the left and right lower limbs were evaluated during gait. Pelvic positions were measured using a three-dimensional spinal diagnostic imaging system (Back Mapper, ABW, Germany). This system provides relatively accurate analysis of shapes, positions, and distortions of the human spine when viewed from the front, rear, bottom, or top of the body. In addition to evaluating the distribution of fat and muscle, this system shows the position of the skeleton in the body to analyze pelvic position (PPO), which provides the lateral slope of the pelvis. The system also shows pelvic torsion (PTO, the degree of torsion of the hipbone), and pelvic rotation (PRO, the degree of torsion of the pelvis on the horizontal plane).

The Qualisys Track Manager (Qualisys AB, Sweden) is a three-dimensional wireless motion analysis system equipped with 6 cameras operating at $100 \mathrm{~Hz} / \mathrm{second}$. This system was used for kinematic analysis of motion in the lower limbs during gait. An L-frame was fixed at the point at which the gait began in the body to set the spatial coordinates. The upward vertical axis, moving direction axis, and horizontal axis were set as $+\mathrm{Z},+\mathrm{Y}$, and $+\mathrm{X}$ axes, respectively. To ensure that the marker was recognized, the space in which movements were performed was defined using a T-wand based on the L-frame. Reflective markers were attached to the sacrum, both thighs, both anterior superior iliac spines, and the sides of the shanks to form a basic frame. The four markers on the thighs and the sides of the shanks were attached in a square to form a three-dimensional space. Markers were also placed on the medial side of the femurs, lateral epicondyles, lateral malleolus, and on both sides of the medial malleolus. In terms of the segments of the subjects' feet, markers were attached to the calcaneal tuberosity and the first and fifth metatarsal heads on the surface of the feet. In addition, markers were placed on the medial side of the femurs, the lateral epicondyles, the lateral malleolus, and on both sides of the medial malleolus to track the three-dimensional movements of the three lower limb joints. These markers were removed before images were taken. A single trained individual attached the markers to reduce the potential for error. Using motion analysis, differences were measured between the left and right range of motion for hip joint flexion, abduction, and external rotation. Differences were also measured for knee joint flexion, abduction, and external rotation, as well as ankle joint dorsiflexion, inversion, and abduction along the $\mathrm{X}, \mathrm{Y}$, and $\mathrm{Z}$ axes $^{11)}$.

The measured data were analyzed using the SPSS 12.0 KO (SPSS, Chicago, IL, USA) statistical program. The collected data were presented as means and standard deviations. Paired t-tests were used to measure the significance of differences before and after the experiment in each group, and independent t-tests determined the significance of differences between the two groups. The level of significance was set to 0.05 .

\section{RESULTS}

The data were compared before and after the experiment in the pelvic adjustment and stretching group. According to the results, the pelvic adjustment group showed a statistically significant change in hip joint flexion (HXD), knee abduction 
Table 1. Comparisons between pre- and post-intervention in each group (mean \pm SD) (unit: degree)

\begin{tabular}{|c|c|c|c|}
\hline Category & Group & Pre-intervention & Post-intervention \\
\hline \multirow{2}{*}{ HXD } & Adjustment group* & $5.75 \pm 2.32$ & $3.00 \pm 2.34$ \\
\hline & Stretching group & $6.18 \pm 4.43$ & $5.04 \pm 2.21$ \\
\hline \multirow{2}{*}{ HYD } & Adjustment group & $3.61 \pm 2.51$ & $2.69 \pm 2.14$ \\
\hline & Stretching group & $3.49 \pm 2.45$ & $3.61 \pm 1.78$ \\
\hline \multirow{2}{*}{ HZD } & Adjustment group & $5.02 \pm 3.84$ & $2.69 \pm 2.14$ \\
\hline & Stretching group & $4.70 \pm 1.94$ & $3.61 \pm 1.78$ \\
\hline \multirow{2}{*}{ KXD } & Adjustment group & $6.75 \pm 5.88$ & $3.60 \pm 365$ \\
\hline & Stretching group & $6.08 \pm 5.50$ & $5.01 \pm 2.03$ \\
\hline \multirow{2}{*}{ KYD } & Adjustment group* & $6.57 \pm 6.14$ & $2.58 \pm 1.84$ \\
\hline & Stretching group & $5.13 \pm 2.56$ & $4.30 \pm 2.56$ \\
\hline \multirow{2}{*}{ KZD } & Adjustment group & $6.56 \pm 5.10$ & $4.31 \pm 3.91$ \\
\hline & Stretching group & $6.67 \pm 3.51$ & $5.33 \pm 4.24$ \\
\hline \multirow{2}{*}{ AXD } & Adjustment group & $5.74 \pm 4.67$ & $4.64 \pm 3.61$ \\
\hline & Stretching group & $6.92 \pm 5.91$ & $5.65 \pm 3.62$ \\
\hline \multirow{2}{*}{ AYD } & Adjustment group & $5.16 \pm 3.95$ & $3.45 \pm 3.01$ \\
\hline & Stretching group & $6.10 \pm 4.29$ & $5.68 \pm 3.40$ \\
\hline \multirow{2}{*}{ AZD } & Adjustment group & $5.94 \pm 6.18$ & $4.57 \pm 2.92$ \\
\hline & Stretching group & $5.26 \pm 2.89$ & $5.57 \pm 4.04$ \\
\hline \multirow{2}{*}{ PPO } & Adjustment group* & $2.52 \pm 1.50$ & $1.58 \pm 0.50$ \\
\hline & Stretching group & $2.41 \pm 1.41$ & $2.05 \pm 0.82$ \\
\hline \multirow{2}{*}{ PTO } & Adjustment group* & $3.64 \pm 2.47$ & $2.11 \pm 0.78$ \\
\hline & Stretching group & $3.47 \pm 2.87$ & $3.05 \pm 1.51$ \\
\hline \multirow{2}{*}{ PRO } & Adjustment group & $2.52 \pm 2.32$ & $1.70 \pm 0.68$ \\
\hline & Stretching group & $2.41 \pm 1.83$ & $2.17 \pm 1.33$ \\
\hline
\end{tabular}

* $\mathrm{p}<0.05$, XD: X-axis difference value between left and right, YD: Y-axis difference value between left and right, ZD: Z-axis difference value between left and right, P: pelvic, H: hip, K: knee, A: ankle, HXD: difference of hip flexion between left and right, HYD: difference of hip abduction between left and right, HZD: difference of hip external rotation between left and right, KXD: difference of knee flexion between left and right, KYD: difference of knee abduction between left and right, KZD: difference of knee external rotation between left and right, AXD: difference of ankle dorsiflexion between left and right, AYD: difference of ankle abduction between left and right, AZD: difference of ankle inversion between left and right, PPO: pelvic position, PTO: pelvic torsion, PRO: pelvic rotation.

(KYD), PPO, and PTO ( $<<0.05)$, while the stretching group showed no statistically significant difference in any of the items $(\mathrm{p}>0.05)$ (Table 1). The differences in the data measured before and after the experiment in the pelvic adjustment group were compared with those in the stretching group. No items showed significant differences between the groups before the experiment $(p>0.05)$. However, HXD, KYD, and PTO were statistically significantly different between the groups after the experiment $(\mathrm{p}<0.05)$, and differences before and after the experiment showed no statistical significance in any of the items $(\mathrm{p}>0.05)($ Table 2$)$.

\section{DISCUSSION}

Normal gait is important to maintain harmonious trunk and pelvic movements. The dynamic balance of trunk muscle activities limits unnecessary energy consumption and effectively reduces false steps that may occur during gait ${ }^{4)}$. Incorrect gait patterns cause body instability and affect breathing, the heart, and balance of various regions of the body. Incorrect gait patterns also lead to excessive energy consumption. This may induce severe fatigue, long-term increases of which can cause malformation of the foot structure due to improper weight distribution ${ }^{6}$. The pelvis is a structure between the hip joint and the lumbosacral region. Many muscles are attached to the pelvis to control the movements of the hip joint and the lumbosacral region. Therefore, the position of the pelvis is the most important element that determines the sagittal alignment and posture in the body ${ }^{15)}$. However, the position of the pelvis may cause mechanical problems to the musculoskeletal system of the lumbar vertebrae, and anterior-posterior sloped movements of the pelvis may affect spinal stability and body alignment. Therefore, proper alignment of the pelvis is an important component of gait. Improper positioning of the pelvis can lead to poor posture ${ }^{16)}$. 
Table 2. Comparisons between the adjustment and stretching group (mean $\pm \mathrm{SD}$ ) (unit: degree)

\begin{tabular}{|c|c|c|c|}
\hline & Category & Adjustment group & Stretching group \\
\hline \multirow{12}{*}{ Pre-intervention } & HXD & $5.75 \pm 2.32$ & $6.18 \pm 4.43$ \\
\hline & HYD & $3.61 \pm 2.51$ & $3.49 \pm 2.45$ \\
\hline & HZD & $5.02 \pm 3.84$ & $4.70 \pm 1.94$ \\
\hline & KXD & $6.75 \pm 5.88$ & $6.08 \pm 5.50$ \\
\hline & KYD & $6.57 \pm 6.14$ & $5.13 \pm 2.56$ \\
\hline & KZD & $6.56 \pm 5.10$ & $6.67 \pm 3.51$ \\
\hline & AXD & $5.74 \pm 4.67$ & $6.92 \pm 5.91$ \\
\hline & AYD & $5.16 \pm 3.95$ & $6.10 \pm 4.29$ \\
\hline & AZD & $5.94 \pm 6.18$ & $5.26 \pm 2.89$ \\
\hline & PPO & $2.52 \pm 1.50$ & $2.41 \pm 1.41$ \\
\hline & PTO & $3.64 \pm 2.47$ & $3.47 \pm 2.87$ \\
\hline & PRO & $2.52 \pm 2.32$ & $2.41 \pm 1.83$ \\
\hline \multirow{12}{*}{ Post-intervention } & HXD* & $3.00 \pm 2.34$ & $5.04 \pm 2.21$ \\
\hline & HYD & $2.69 \pm 2.14$ & $3.61 \pm 1.78$ \\
\hline & HZD & $2.69 \pm 2.14$ & $3.61 \pm 1.78$ \\
\hline & KXD & $3.60 \pm 365$ & $5.01 \pm 2.03$ \\
\hline & KYD* & $2.58 \pm 1.84$ & $4.30 \pm 2.56$ \\
\hline & KZD & $4.31 \pm 3.91$ & $5.33 \pm 4.24$ \\
\hline & AXD & $4.64 \pm 3.61$ & $5.65 \pm 3.62$ \\
\hline & AYD & $3.45 \pm 3.01$ & $5.68 \pm 3.40$ \\
\hline & AZD & $4.57 \pm 2.92$ & $5.57 \pm 4.04$ \\
\hline & PPO & $1.58 \pm 0.50$ & $2.05 \pm 0.82$ \\
\hline & PTO* & $2.11 \pm 0.78$ & $3.05 \pm 1.51$ \\
\hline & PRO & $1.70 \pm 0.68$ & $2.17 \pm 1.33$ \\
\hline \multirow{12}{*}{$\begin{array}{l}\text { Change between pre- and } \\
\text { post-intervention }\end{array}$} & HXD & $2.74 \pm 3.94$ & $1.14 \pm 5.02$ \\
\hline & HYD & $0.92 \pm 3.02$ & $0.13 \pm 3.28$ \\
\hline & HZD & $2.32 \pm 5.01$ & $1.08 \pm 2.21$ \\
\hline & KXD & $3.15 \pm 6.04$ & $1.06 \pm 6.66$ \\
\hline & KYD & $4.16 \pm 6.34$ & $0.83 \pm 5.68$ \\
\hline & KZD & $2.24 \pm 6.12$ & $1.34 \pm 4.96$ \\
\hline & AXD & $1.09 \pm 5.66$ & $1.27 \pm 6.34$ \\
\hline & AYD & $1.71 \pm 5.71$ & $0.42 \pm 4.40$ \\
\hline & AZD & $1.37 \pm 6.53$ & $0.31 \pm 4.59$ \\
\hline & PPO & $0.94 \pm 1.63$ & $0.35 \pm 1.83$ \\
\hline & PTO & $1.52 \pm 2.52$ & $0.41 \pm 3.72$ \\
\hline & PRO & $0.82 \pm 2.57$ & $0.23 \pm 1.48$ \\
\hline
\end{tabular}

$* \mathrm{p}<0.05$

Normal positions and stability of the pelvis are important for balanced gait. Although many previous studies have used three-dimensional gait analysis to implement diverse interventions in hemiplegia ${ }^{17)}$ or cerebral palsy patients ${ }^{18)}$, few studies have analyzed the gait of normal adults after pelvic adjustment. Therefore, this study was designed to examine the effects of pelvic adjustment in normal adults on the position of the pelvis and lower limb joint angles during gait.

After pelvic adjustment was applied using the Gonstead technique, lower limb joint angles during gait were measured using the Qualisys Track Manager. According to the results, differences between left and right hip flexion angles and between left and right knee valgus angles significantly decreased in the experimental group. Pelvic postures were analyzed using a back mapper. The results showed that the lateral slope of the pelvis and hipbone torsion decreased after pelvic adjustment.

In terms of previous studies related to pelvic adjustment, Park et al. reported an improvement in the balance of 20 elderly men through pelvic adjustment using the Gonstead technique ${ }^{19)}$. Gong et al. reported that unequal functional leg length decreased following pelvic adjustment using the Gonstead technique. This change in leg length inequality also reduced foot pressure differences ${ }^{13}$. In another study related to the pelvis, Cho and Jun selected 30 female university students and randomly assigned them to a pelvic adjustment and a stretching group, each of which contained 15 subjects. Cho and Jun's 
study, the purpose of which was to analyze gait and differences in step length, stance phase, swing phase, single support, and step time between the groups, found marked decreases in these elements following pelvic adjustment ${ }^{10)}$. Cho reported that pelvic adjustment led to statistically significant improvements in trunk imbalance, pelvic position, pelvic torsion, and the position of the scapulae ${ }^{9}$.

Previous studies suggesting that pelvic adjustment using the Gonstead technique reduced differences in leg lengths, improved balance, reduced foot pressure differences, reduced differences in gait variables (e.g., step length), and improved pelvic postures (e.g., pelvic positions) are consistent with the results found in the present study. The findings of this study indicate improved gait quality using pelvic adjustment to correct pelvic postures, reduced differences between left and right hip flexion angles, and minimized differences between left and right knee valgus angles. These results were achieved because pelvic adjustment affected balance and gait variables. The use of pelvic adjustment in clinics to improve pelvic postures and gait quality is therefore recommended.

\section{ACKNOWLEDGEMENT}

This research was supported by the Korea Nazarene University Research Grants in 2016.

\section{REFERENCES}

1) Perry J: Gait analysis: Normal and pathological function. New Jersey: Slack, 1992.

2) Vaughan CL, Davis BL, O'Connor JC: Dynamics of human gait, 2nd ed. South Africa: Kiboho Publishers, 1992.

3) Eckert H: Motor development. Indianapolis: Benchaak Press, 1987.

4) White SG, McNair PJ: Abdominal and erector spinae muscle activity during gait: the use of cluster analysis to identify patterns of activity. Clin Biomech (Bristol, Avon), 2002, 17: 177-184. [Medline] [CrossRef]

5) Galley PM, Foster A: Functional evaluation of normal and pathological knees during gait. Arch Phys Med Rehabil, 1987, 57: 571-577.

6) Bowker P, Messenger N: The measurement of gait. Clin Rehabil, 1988, 2: 89-97.

7) Liebenson C: Spinal stabilization training: the transverse abdominus. J Body w and Mov Ther, 1998, 2: $218-223$. [CrossRef]

8) García-Vaquero MP, Moreside JM, Brontons-Gil E, et al.: Trunk muscle activation during stabilization exercises with single and double leg support. J Electromyogr Kinesiol, 2012, 22: 398-406. [Medline] [CrossRef]

9) Cho M: The influence of pelvic adjustment on the posture of female university students. J Phys Ther Sci, 2013, 25: 785-787. [Medline] [CrossRef]

10) Cho M, Jun I: Effects of pelvic adjustment on female university students' gait variables. J Phys Ther Sci, 2014, 26: 759-762. [Medline] [CrossRef]

11) Cho M: Correlations among pelvic positions and differences in lower extremity joint angles during walking in female university students. J Phys Ther Sci, 2015, 27: 1941-1944. [Medline] [CrossRef]

12) Cooperstein R: Gonstead Chiropractic Technique (GCT). J Chiropr Med, 2003, 2: 16-24. [Medline] [CrossRef]

13) Gong WT, Ro HL, Park GD, et al.: The influence of pelvic adjustment on functional leg length inequality and foot pressure. J Phys Ther Sci, 2011, 23: 17-19. [CrossRef]

14) Gong W: The influence of pelvic adjustment on vertical jump height in female university students with functional leg length inequality. J Phys Ther Sci, 2015, 27: 251-253. [Medline] [CrossRef]

15) Vaz G, Roussouly P, Berthonnaud E, et al.: Sagittal morphology and equilibrium of pelvis and spine. Eur Spine J, 2002, 11: 80-87. [Medline] [CrossRef]

16) Walsh M, Connolly P, Jenkinson A, et al.: Leg length discrepancy—an experimental study of compensatory changes in three dimensions using gait analysis. Gait Posture, 2000, 12: 156-161. [Medline] [CrossRef]

17) Defrin R, Ben Benyamin S, Aldubi RD, et al.: Conservative correction of leg-length discrepancies of $10 \mathrm{~mm}$ or less for the relief of chronic low back pain. Arch Phys Med Rehabil, 2005, 86: 2075-2080. [Medline] [CrossRef]

18) Bonnyaud C, Pradon D, Zory R, et al.: Effects of a gait training session combined with a mass on the non-paretic lower limb on locomotion of hemiparetic patients: a randomized controlled clinical trial. Gait Posture, 2013, 37: 627-630. [Medline] [CrossRef]

19) Park GD, Ju SB, Jang HJ: The effect of pelvic adjustment on the stability of elderly men. J Phys Ther Sci, 2011, 23: 937-939. [CrossRef] 